\title{
Research of New Method for Removal thin Cloud and Fog of the Remote Sensing Images
}

\author{
JIANG Xingfang \\ State Key Laboratory of Satellite Ocean Environment \\ Dynamics \\ Second Institute of Oceanography, State Oceanic \\ Administration \\ Hangzhou, China \\ xfjiang@jpu.edu.cn
}

\author{
MA Wei \\ School of mathematics and Physics \\ Jiangsu Polytechnic University \\ Changzhou 213164, China \\ mawei@smail.jpu.edu.cn
}

\begin{abstract}
For the questions of obfuscation in Google earth remote sensing images, the new method had been pointed out. The cause of obfuscation was the interference of thin cloud and fog. The new method was combination of the advanced multi-scale Retinex (AMSR) and the method of Homomorphic Filter. The results were shown the new method was effectual for removing the thin cloud and fog from the remote sensing images in Google earth. The AMSR included three steps. The first step was to get the complementary color image for the remote sensing images in Google Earth with thin cloud and fog. Next, the enhanced images were stretched with MSR in different $k$ Multiples standard deviation near the average brightness. Finally, the new complementary color image of the enhanced images had been got. The criterion of information entropy was as the image quality criterion. The conclusion shows that the new images were high quality. The new images handled by the method of Homomorphic Filter after enhanced by AMSR. The parameter of Homomorphic Filter was best in $0.9 \pm 0.1$ and the remote sensing images handled by new method have largest information entropy.
\end{abstract}

Keywords- Information optics; advanced multi-scale Retinex; Homomorphic Filter; cloud and fog

\section{INTRODUCTION}

We can find the regions are obfuscation in the remote sensing images of Google Earth web because of there are cloud and fog. The general methods for removing cloud and fog from the remote sensing images were the Method of Multi-optical Spectrum ${ }^{[1]}$, the Interpolation Method of Multiimage, the Fusion Method of Multi-source ${ }^{[2]}$, the Method of Homomorphic Filter ${ }^{[3,4]}$, and the Removing Cloud Method from Single-image ${ }^{[5,6]}$. Method of Multi-optical Spectrum was based on the sensitive band in the sensor to remove cloud and fog from remote sensing images. The Interpolation Method of Multi-image was based on many images in different time in same site to remove cloud and fog. The Fusion Method of Multi-source was based on the information of multi-sensor to remove cloud and fog. The Method of Homomorphic Filter was based on the model of intensity of illumination and reflectivity to remove cloud and fog. The result was the production of illumination and reflectivity. The process included FFT and high pass filter. The information in the dark regions was protruded. This paper was another new method after references [5-6]. The new method was combination of the advanced multi-scale Retinex (AMSR) and the method of Homomorphic Filter to remove cloud and fog.

\section{ADVANCED MUlTi-SCALE RETINEX}

The idea of Retinex was figured by E. Land on 30, December, 1963 in Ohio. The Retinex was combination of retina and cortex. The idea of Retinex was based on the intensity of incident light $E$ and reflectance $R, L=E \cdot R$. Where $E$ decided the dynamic range for each pixel and $R$ decided the inscape for images. The purpose of Retinex was to subtract the incident light in logarithmic domain. Because of the logarithmic domain was near the characteristics of the percipience ability of the eyes and the operation was very simple.

The Multi-scale Retinex expression was figured by D. J. Jobson, Z. Rahman, G. A. Woodell et al. ${ }^{[8-13]}$

$$
R_{l}(i, j)=\sum_{n=1}^{N} W_{n}\left\{\log I_{l}(i, j)-\log \left[F_{n}(i, j) * I_{l}(i, j)\right]\right\}
$$

Where, $l=1,2,3$ expressed red, green, and blue respectively. $W_{n}$ was weight for $F_{n} . F_{n}$ was center / surrounding function

$$
F_{n}(i, j)=A \exp \left\{-\frac{i^{2}+j^{2}}{2 \sigma_{n}^{2}}\right\}
$$

Where $\sigma_{n}$ was standard deviation in Guamanian function. $A$ was normalization constant.

$$
A=\frac{1}{\sum_{j} \sum_{i} F_{n}(i, j)}
$$

The interesting point was the multi-scale Retinex backed to single-scale Retinex when $n=1$ and $W_{1}=1$.

The AMSR had two levels. The first one was the redundancy of the enhanced images had been considered. The enhanced images had been stretched with the $k$ Multiples standard deviation near the average of brightness based on proper distributions. Another one was based on the characteristics of the cloud and fog. The regions were bright because there were cloud and fog in remote sensing images.

\section{OBTAINING REMOTE SENSING IMAGES AND REMOVING CLOUD AND FOG FROM THEM}

We can get many remote sensing images in those there are many regions are covered cloud and fog in remote sensing images from Google earth wet. The information in the regions could not be resolved and the images were 
shown as the first column in Table 1. Image1 was covered by fog and cloud. Image 2 and Image 3 were covered by fog. Image 4 was covered by cloud in a small region. For removing thin cloud and fog in Image1 the AMSR was used in three steps. The first step was to select the complementary color image of the original image and the bright regions of cloud and fog were turned to dark regions. The second step was to apply the method of the AMSR algorithm to enhance the information in dark region, and the range of $k$ was from 1 to 3 with the step as 0.1 . The last step was to select the complementary color image of the enhanced image by AMSR. The other three images shown in table 1 were enhanced as same method.
The criterion of information entropy was as the image quality criterion for four group's images of four original images. The maximum entropies for each group had been got. The maximum entropies were shown in table 2 . The curves were shown in fig. 1(a). The relation between the average entropy with $k$ was shown in fig. 1(b). The images of the maximum entropy for each group were shown in the second column in Table 1.

The information entropy was defined ${ }^{[4]}$ as

$$
\text { Entropy }=-\sum_{i=0}^{255} P_{i} \log _{2} P_{i}
$$

Where $P_{i}$ expressed the ratio of the pixel number of gray value was $i$ to the whole pixel number.

Tabel 1 Comparing of the enhanced remote sensing images with original images

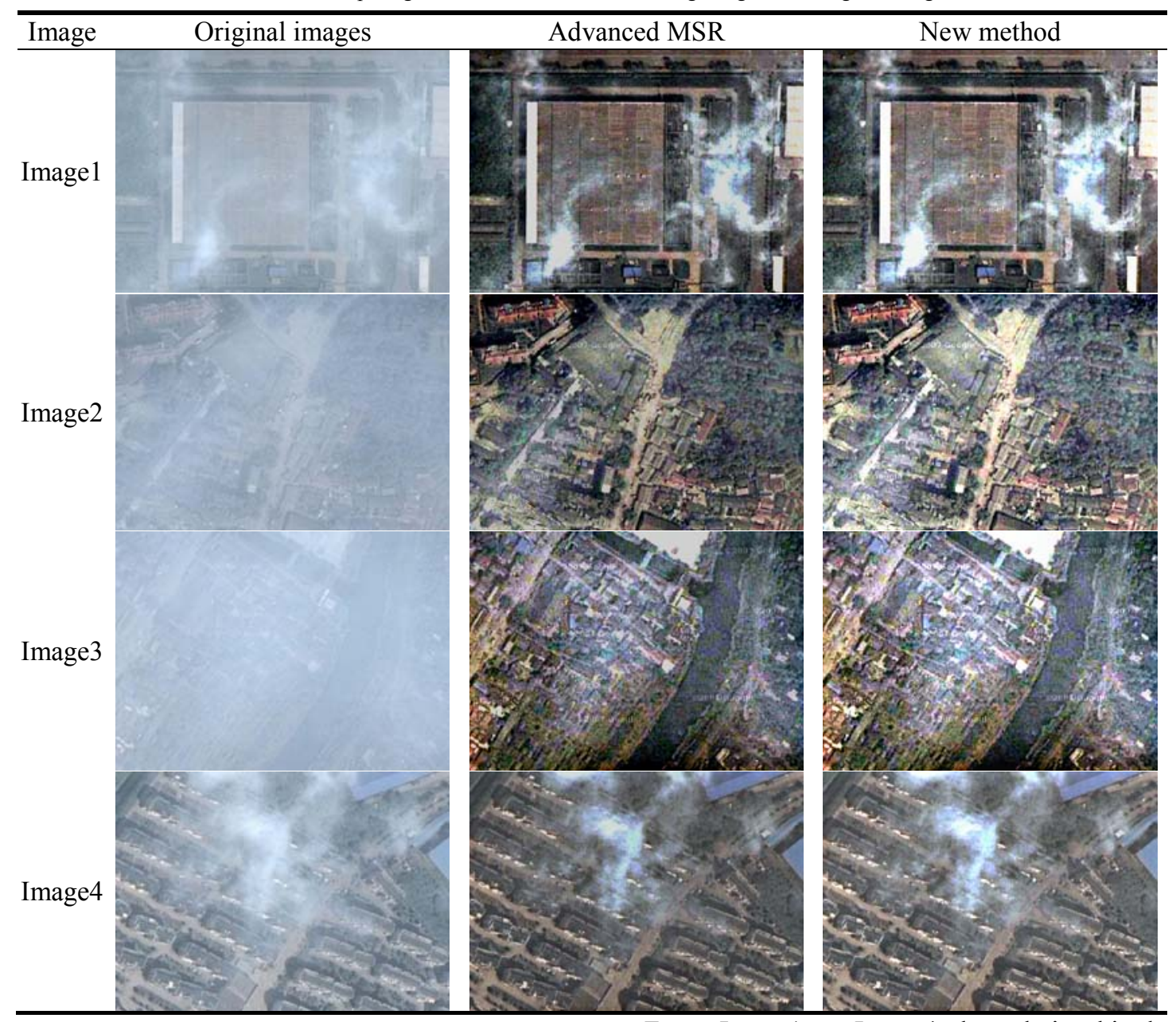

From Image1 to Image4 the relationship between the

The new method was that the method of Homomorphic Filter was used for the remote sensing images enhanced by AMSR with maximum entropy. The parameter of Homomorphic Filter was choose in the range $0.9 \pm 0.1$ and the handled remote sensing images were best because the entropy was larger than that of the remote sensing images enhanced by AMSR directly. entropies with $n$ s was shown as the part program of Matlab.

$n=\left[\begin{array}{lllllllllllll}0.3 & 0.4 & 0.5 & 0.6 & 0.7 & 0.8 & 0.9 & 1 & 1.1 & 1.2 & 1.3 & 1.4 & 1.5\end{array}\right]$;

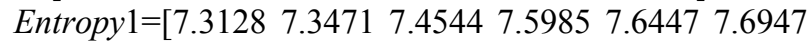

7.7293 7.7386 7.7246 7.6872 7.6281 7.5545 7.4789];

Entropy2 $=\left[\begin{array}{llllll}7.3139 & 7.3377 & 7.4740 & 7.6760 & 7.7068 & 7.7240\end{array}\right.$

$7.72197 .69857 .66107 .60457 .53657 .46197 .3931]$;

Entropy3 $=\left[\begin{array}{llllll}7.3523 & 7.3784 & 7.5063 & 7.6451 & 7.6591 & 7.6794\end{array}\right.$

7.67897 .65267 .6027 7.5396 7.4725 7.4040 7.3373]; 
Entropy4=[7.3121 7.35067 .48807 .67607 .71217 .7364 7.7405 7.7252 7.6930 7.6437 7.5806 7.5183 7.4647];

The quadratic fitting curves of handled remote sensing images by new method were shown in Fig. 2. The fitting curves said respectively as follow

$$
\begin{aligned}
& \text { Entropy } 2=-1.1 n^{2}+2.1 n+6.8, \\
& \text { Entropy } 3=-0.98 n^{2}+1.7 n+6.9, \\
& \text { Entropy } 4=-1.1 n^{2}+2 n+6.8 .
\end{aligned}
$$

\begin{tabular}{|c|c|c|c|c|c|c|c|}
\hline image & & & & $k$ & & & \\
\hline \multirow{6}{*}{ Image1 } & 1.0 & 1.1 & 1.2 & 1.3 & 1.4 & 1.5 & 1.6 \\
\hline & 7.0895 & 7.2039 & 7.2769 & 7.3049 & 7.3028 & 7.2903 & 7.2628 \\
\hline & 1.7 & 1.8 & 1.9 & 2.0 & 2.1 & 2.2 & 2.3 \\
\hline & 7.2294 & 7.1880 & 7.1524 & 7.1134 & 7.0750 & 7.0419 & 7.0063 \\
\hline & 2.4 & 2.5 & 2.6 & 2.7 & 2.8 & 2.9 & 3.0 \\
\hline & 6.9708 & 6.9366 & 6.8993 & 6.8667 & 6.8347 & 6.8021 & 6.7669 \\
\hline \multirow{6}{*}{ Image 2} & 1.0 & 1.1 & 1.2 & 1.3 & 1.4 & 1.5 & 1.6 \\
\hline & 6.4906 & 6.7037 & 6.8687 & 7.0051 & 7.1037 & 7.1703 & 7.2317 \\
\hline & 1.7 & 1.8 & 1.9 & 2.0 & 2.1 & 2.2 & 2.3 \\
\hline & 7.2664 & 7.2803 & 7.2934 & 7.2894 & 7.2798 & 7.2639 & 7.2414 \\
\hline & 2.4 & 2.5 & 2.6 & 2.7 & 2.8 & 2.9 & 3.0 \\
\hline & 7.2155 & 7.1856 & 7.1524 & 7.1194 & 7.0845 & 7.0492 & 7.0153 \\
\hline \multirow{6}{*}{ Image 3 } & 1.0 & 1.1 & 1.2 & 1.3 & 1.4 & 1.5 & 1.6 \\
\hline & 6.9130 & 7.0676 & 7.1747 & 7.2548 & 7.2994 & 7.3193 & 7.3276 \\
\hline & 1.7 & 1.8 & 1.9 & 2.0 & 2.1 & 2.2 & 2.3 \\
\hline & 7.3209 & 7.3024 & 7.2798 & 7.2519 & 7.2200 & 7.1855 & 7.1489 \\
\hline & 2.4 & 2.5 & 2.6 & 2.7 & 2.8 & 2.9 & 3.0 \\
\hline & 7.1148 & 7.0795 & 7.0432 & 7.0075 & 6.9734 & 6.9381 & 6.9045 \\
\hline \multirow{6}{*}{ Image4 } & 1.0 & 1.1 & 1.2 & 1.3 & 1.4 & 1.5 & 1.6 \\
\hline & 7.1535 & 7.2550 & 7.2992 & 7.3037 & 7.2817 & 7.2504 & 7.2143 \\
\hline & 1.7 & 1.8 & 1.9 & 2.0 & 2.1 & 2.2 & 2.3 \\
\hline & 7.1725 & 7.1298 & 7.0876 & 7.0457 & 7.0013 & 6.9598 & 6.9171 \\
\hline & 2.4 & 2.5 & 2.6 & 2.7 & 2.8 & 2.9 & 3.0 \\
\hline & 6.8771 & 6.8362 & 6.7957 & 6.7557 & 6.7170 & 6.6790 & 6.6416 \\
\hline \multirow{6}{*}{ average } & 1.0 & 1.1 & 1.2 & 1.3 & 1.4 & 1.5 & 1.6 \\
\hline & 7.1535 & 7.2550 & 7.2992 & 7.3037 & 7.2817 & 7.2504 & 7.2143 \\
\hline & 1.7 & 1.8 & 1.9 & 2.0 & 2.1 & 2.2 & 2.3 \\
\hline & 7.1725 & 7.1298 & 7.0876 & 7.0457 & 7.0013 & 6.9598 & 6.9171 \\
\hline & 2.4 & 2.5 & 2.6 & 2.7 & 2.8 & 2.9 & 3.0 \\
\hline & 6.8771 & 6.8362 & 6.7957 & 6.7557 & 6.7170 & 6.6790 & 6.6416 \\
\hline
\end{tabular}

Entropy $1=-0.97 n^{2}+1.9 n+6.8$,

Tabel 2 Relation of entropy $\sim k$ for the handled images by AMSR

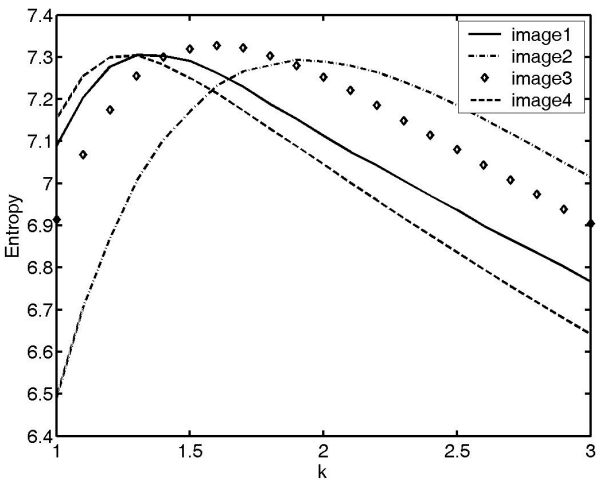

(a) Entropy $\sim \mathrm{k}$ for four images

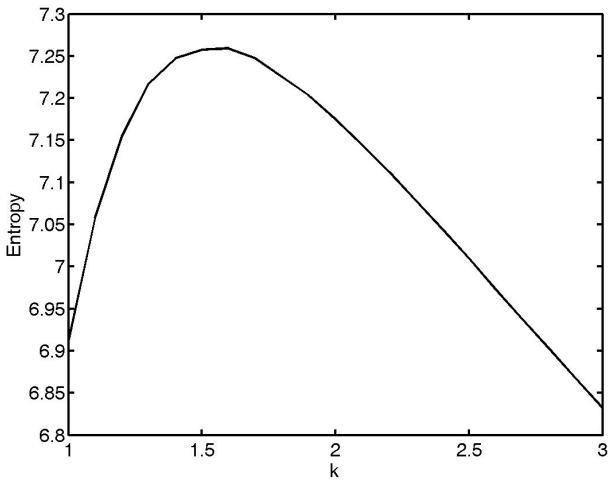

(b) Average entropy $\sim \mathrm{k}$

Fig. 1 The curves bath entropy and $\mathrm{k}$ 


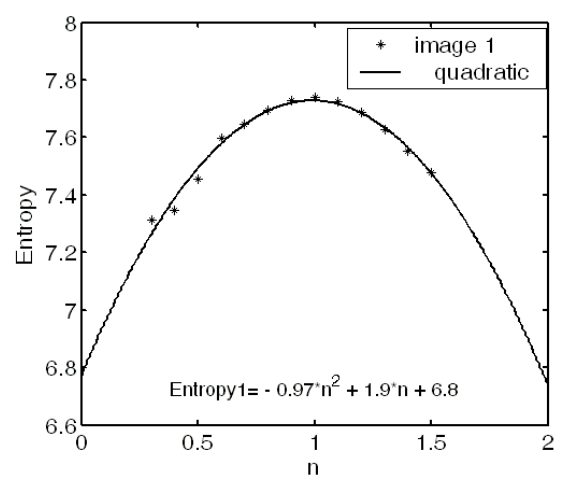

(a) for Image1

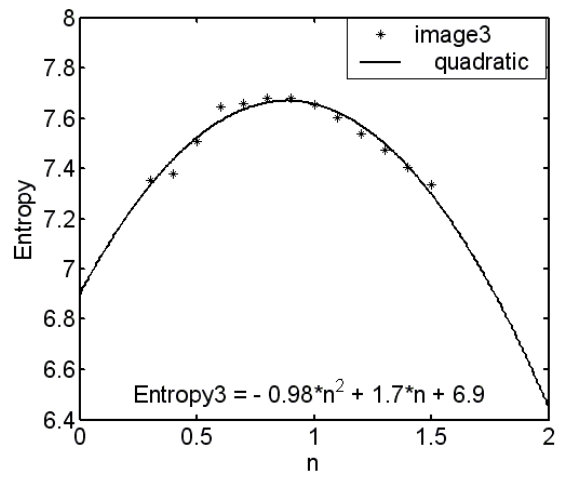

(a) for Image3

Fig. 2 The quadratic curves of Entropy $\sim \mathrm{n}$ after high pass filtered by the method of homomorphism filter

\section{CONCLUSION}

For the remote sensing images from Image1 to Image4, The AMSR algorithm is good method for removing cloud and fog. The enhanced remote sensing images have maximum entropy with $k=1.6 \pm 0.5$ and the thin cloud and fog can be removed. The new method is that the Homomorphic Filter can be used for the enhanced remote sensing images by AMSR with maximum entropy. The conclusion we can get the parameter $n$ of Homomorphism Filter is in the range of $0.9 \pm 0.1$. The remote sensing images handled by new method have larger entropy than the enhanced images by AMSR, such as 7.73 is larger than 7.30.

\section{ACKNOWLEDGMENT}

This paper authors express their sincere thanks to the reviewer for suggestions and comments. This work was supported by the open issues of State Key laboratory of Satellite Ocean Environment Dynamics (No. SOED0912) and the technology Fund of Jiangsu Polytechnic University (ZMF08020003).

\section{REFERENCES}

[1] Li Xiao-chun, WANG Yong, Chen Jing, "Detection and removal of clouds and their shadows from multi-spectral image". Journal of Astronautics. Vol. 25, May. 2004, pp. 555-559 (in Chinese).

[2] S. Gabarda, G. Cristobal, "Cloud civering denoising through image fusion”. ScienceDirect. Vol. 25, 2007, pp. 523-530.

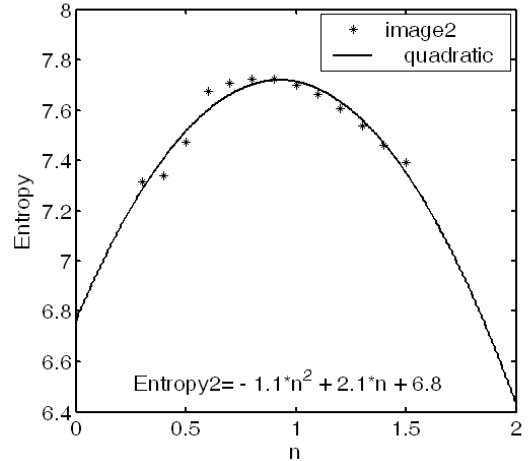

(b) for Image2

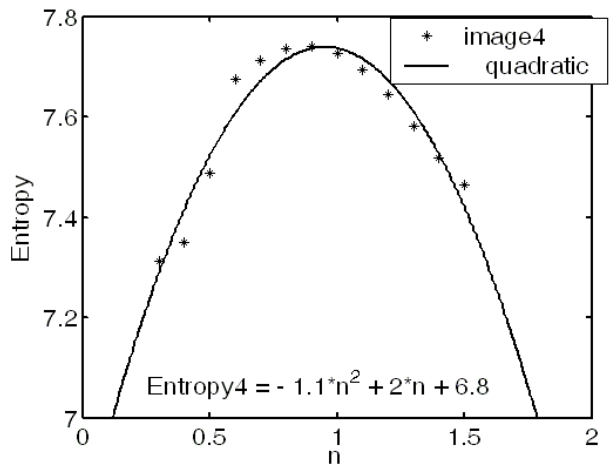

(b) for Image 4

[3] C. Feng, J. W. Ma, Q. Dai, et al. "An improved method for rapid removal of thin cloud in remote sensing images". Remote sensing for land \& resources. Vol. 62, April, 2004, pp. 1-5 (in Chinese).

[4] H. M. Xie , Q. He, N. Zhang, et al. "The improved homomorphic filter algorithm for removing cloud of remote sensing image based on the second exploiture of ERDAS tool". Journal of Beijing Normal University. Vol. 41, February, 2005, pp. 150-153 (in Chinese).

[5] X. F. Jiang, G. W. Pan, C. K. Tao. "Application of Huygens secondarywave method in eliminating cloud and its shadow for remote sensing image". Journal of applied optics. Vol. 28, February, 2007, pp. 165168 (in Chinese).

[6] X. F. Jiang, G. W. Pan, C. K. Tao. "Cloud elmination method in remote sensing image based on spline curve". Laser Technology. Vol. 31, June, 2007, pp. 581-583 (in Chinese).

[7] J. J. McCann. "Retinex at 40". Journal of Electronic Imaging, vol. 13, January, 2004, pp. 6-7.

[8] J. J. McCann. "Capturing a Black Cat in Shade: the Past and Present of Retinex Color Appearance Models". SPIE. Vol. 4662, 2002, pp.331340.

[9] A. Rizzr, D. Gadia, D. Marini. "Spectral Information and Spatial Color Computation”. SPIE. Vol. 5667, 2005, pp. 22-27.

[10] E. Land, J. J. McCann. "Lightness and Retinex Theory". Journal of the Optical Society of America. Vol. 61, January, 1971, pp. 1-11.

[11] D. J. Jobson, Z. Rahman, G. A. Woodell. "Properties and Performance of a Center/Surround Retinex". IEEE TRANSACTIONS ON IMAGE PROCESSING. Vol. 6, March, 1997, pp. 451-462.

[12] Z. Rahman, D. J. Jobson, G. A. Woodell. “A Multiscale Retinex for Bridging the Gap between Color Images and the Human Observation of Senses". IEEE Transactions on Image Processing. Vol. 1, July, 1997, pp. 965-976.

[13] B. Thompson, Z. Rahman, S. Park. "Retinex Preprocessing for Improved Multi-spectral Image Classification”. SPIE. Vol. 3716, 1999, pp. 29-39. 